\title{
Les restructurations, entre opportunités et contraintes :
}

Des cadres, ingénieurs et techniciens en reconversion créent leur entreprise dans le secteur des énergies renouvelables

Restructuring industry: former high skilled workers create their own business in renewable energy sector

\section{Annie Lamanthe}

\section{(2) OpenEdition}

Journals

\section{Édition électronique}

URL : http://journals.openedition.org/travailemploi/6344

DOI : 10.4000/travailemploi.6344

ISSN : 1775-416X

Éditeur

DARES - Ministère du Travail

\section{Édition imprimée}

Date de publication : 1 avril 2014

Pagination : 69-84

ISSN : 0224-4365

\section{Référence électronique}

Annie Lamanthe, «Les restructurations, entre opportunités et contraintes : », Travail et Emploi [En

ligne], 138 | avril-juin 2014, mis en ligne le 01 avril 2016, consulté le 19 avril 2019. URL : http:// journals.openedition.org/travailemploi/6344; DOI : 10.4000/travailemploi.6344 


\title{
Les restructurations, entre opportunités et contraintes : des cadres, ingénieurs et techniciens en reconversion créent leur entreprise dans le secteur des énergies renouvelables
}

\author{
Annie Lamanthe $\left.{ }^{*}\right)$
}

L'article est consacré à la création d'entreprises par d'anciens cadres, ingénieurs et techniciens, principalement issus d'entreprises multinationales de haute technologie. Ces entreprises connaissent des mouvements de restructuration qui se déroulent pratiquement de façon continue et font aujourd'hui partie intégrante de leurs stratégies. Dans ce cadre, les salariés concernés se sont saisi d'une double opportunité : ils ont quitté les postes qu'ils occupaient dans les marchés internes de ces entreprises en bénéficiant des dispositifs visant à faciliter les départs; et ils ont créé leur propre entreprise dans l'installation d'équipements en énergie solaire grâce aux perspectives offertes par les problématiques environnementales. Basé sur des recherches portant sur les dynamiques économiques territoriales en région Provence-Alpes-Côte-d'Azur, l'article s'intéresse plus particulièrement aux caractéristiques, facteurs et ressorts des mobilités de ces ex-salariés. Deux profils d'entrepreneurs sont identifiés : les " solaristes》 et les «néo-artisans》.

Depuis les années 1980, les restructurations d'entreprises ont pris une ampleur nouvelle. Caractérisées dans le passé par des crises brutales conduisant à la fermeture d'établissements ou à des «remaniements massifs de secteurs entiers» (RAVEYre, 2005), longtemps associées aux destinées de la «vieille industrie» en perte de vitesse, aux territoires de monoactivité et aux problématiques de reconversion d'ouvriers non qualifiés dans des bassins d'emploi sinistrés, elles se déploient aujourd'hui au travers de nouvelles figures. Ce sont désormais des processus qui se déroulent en continu parce qu'ils font partie des stratégies mêmes des entreprises : remodelages permanents des contours de l'entreprise (filialisation, sous-traitance en cascade, etc.), remaniements structurels internes et externes (réduction des effectifs, recomposition de la main-d'œuvre, réorganisation du travail, etc.). Ils touchent ainsi directement des salariés hautement qualifiés et les grandes entreprises multinationales des industries et services les plus en pointe, sur lesquelles on comptait pourtant pour compenser les pertes d'emplois dans les activités traditionnelles. Devenues moins spectaculaires et plus complexes, les restructurations sont aujourd'hui multiformes et difficiles à cerner, tant du point de vue statistique que juridique (Moreau, 2007; Beaujolin-Bellet

(*) Aix-Marseille Université - Laboratoire d'économie et de sociologie du travail (Lest); annie.lamanthe@univ-amu.fr. Les enquêtes sur lesquelles s'appuie cet article ont été réalisées en collaboration avec Hubert Amarillo, chercheur associé au Lest. et al., 2012). La gestion de leurs conséquences sur l'emploi a également changé : les recours des entreprises aux dispositifs incitant aux départs volontaires se multiplient, privilégiant ainsi les traitements individualisés plutôt que les licenciements massifs. Du côté des salariés, alors que ceux qui s'opposent aux décisions des directions et cherchent à négocier collectivement les conditions de leur licenciement s'engagent dans des conflits longs et parfois violents, d'autres cèdent aux «attraits» du départ volontaire. $\mathrm{Ce}$ mouvement continu de restructuration place les salariés dans un état durable d'incertitude et d'instabilité et a pour effets de nouvelles formes de pénibilité et de vulnérabilité (RAVEYRE, 2007), qui conduisent certains d'entre eux à souhaiter quitter leur emploi pour créer leur propre entreprise.

Ce double processus - de sortie d'une grande entreprise et d'entrée dans l'entrepreneuriat contribue au renouvellement des tissus productifs sur les territoires. Différentes recherches conduites dans la région Provence-Alpes-Côte-d'Azur ont fait ressortir qu'il est directement à l'origine des dynamiques économiques et entrepreneuriales à l'œuvre dans certains segments de l'activité (Amarillo, 2009; Lamanthe, 2011). C'est notamment le cas dans de nouveaux domaines d'activité, comme ceux qui ont émergé avec le développement des problématiques énergétiques. Ces travaux montrent en effet que, parmi les différents types d'entreprises s'étant positionnées sur le marché de l'installation d'équipements en énergies renouvelables, un nombre non négligeable a 
été créé par d'anciens cadres, ingénieurs et techniciens des industries des hautes technologies et des services qualifiés (Amarillo, LAmanthe, 2011). Ces trajectoires s'inscrivent dans une stratégie de type «exit» (Hirschman, 1972) dans laquelle s'engagent des salariés désireux de quitter le monde des grandes entreprises tout en se saisissant d'une double opportunité : d'une part, les ressources offertes par les dispositifs déployés dans le cadre des restructurations; d'autre part, les perspectives de marché ouvertes par la montée des préoccupations environnementales dans la société.

Dans une étude plus récente sur l'ancien bassin minier de Provence(1) (Gallenga, Lamanthe, 2012), des entretiens ont été réalisés avec certains de ces néo-entrepreneurs (population essentiellement masculine), repérés parmi les dirigeants d'entreprises installatrices d'équipement en énergies renouvelables implantées sur ce territoire. Par leur récit, ils éclairent les caractéristiques d'une mobilité qui les a conduits du statut de salarié à celui d'indépendant ainsi que les contextes et conditions qui y ont présidé. S'appuyant sur une conception des trajectoires individuelles - ou parcours - entendues comme une combinaison entre dimensions personnelles et facteurs institutionnels ${ }^{(2)}$, l'article fait ressortir comment les implications des restructurations sur ces ex-salariés se sont articulées avec un ensemble d'autres éléments pour construire leurs mobilités. On peut en effet considérer que celles-ci se situent à l'intersection entre, d'une part, des expériences, motivations et stratégies individuelles (ce sera l'objet de la première partie) et, de l'autre, les reconfigurations du système productif, les perspectives d'activité liées à la transition énergétique et les politiques publiques développées dans ces deux domaines (deuxième partie). Pour autant, les trajectoires ne sont pas toutes identiques et l'on peut distinguer deux profils parmi les néo-entrepreneurs rencontrés : les «solaristes» et les «néo-artisans». Cette distinction s'explique à la fois par les ressources en leur possession et leurs capacités à se saisir de nouvelles ressources et opportunités. On observera donc ici comment leurs capitaux scolaires, leurs trajectoires anté-

(1) Le bassin minier de Provence correspond au périmètre d'un territoire en reconversion qui regroupe dix-sept communes à l'est du département des Bouches-du-Rhône, entre Aix-enProvence, Marseille et le département du Var.

(2) Référence est faite ici à la notion de parcours telle que la propose Bénédicte ZimmermanN (2011). Le parcours consiste ainsi en un enchevêtrement de différents éléments d'ordre privé, social et personnel, organisationnel et institutionnel, supposant l'existence de ressources et d'opportunités pour les individus; il n'est pas réductible à des choix personnels car il est également fait de non-choix et de contraintes, et se déploie «dans des espaces dont la structuration collective contribue à façonner les possibles et la latitude de choix individuels» (p. 85). On insiste aussi par là sur l'importance des variables subjectives dans les bifurcations de carrière, comme le suggère Sophie PochIC (2001) à travers l'intérêt porté au projet professionnel chez les cadres au chômage. rieures et les réseaux dans lesquels ils s'insèrent contribuent à des positionnements économiques et des constructions identitaires différenciés (troisième partie).

\section{De la grande entreprise à l'entrepreneuriat}

L'étude parue en 2012 sur les entreprises installatrices d'équipements en énergies renouvelables implantées sur le bassin minier de Provence permet d'apporter des éléments éclairant les dynamiques de mobilité professionnelle d'anciens cadres, ingénieurs et techniciens pris dans les processus et stratégies de restructuration des grandes entreprises dans lesquelles ils travaillaient et où ils ont accompli une partie de leur carrière. Les entretiens font ressortir que la nouvelle modalité de restructurations à laquelle ils ont été confrontés leur a servi tout à la fois de motivation et de soutien à la mobilité : d'un côté, il s'agit pour eux de quitter l'entreprise pour fuir un avenir incertain et un climat insatisfaisant; de l'autre, ils peuvent bénéficier des opportunités et ressources offertes par les dispositifs accompagnant les réductions d'effectifs. En contrepoint, et rétrospectivement, ceux qui ont été les porteurs de projets de création d'entreprise déclarent qu'ils ont ainsi pu combiner une double perspective : celle de réalisation d'un désir d'autonomie et, paradoxalement, du moins à première vue, celle de recherche de sécurité et de stabilité, qui s'est saisie de l'ouverture de nouveaux marchés dans le domaine des énergies renouvelables.

\section{Des mobilités qui servent une dynamique de création d'entreprise}

Nous avons identifié quarante-quatre entreprises exerçant une activité d'installation d'équipements en énergies renouvelables sur les communes du bassin minier de Provence. Parmi celles que nous avons pu contacter, nous avons réalisé un entretien avec le dirigeant de certaines (voir encadré). Onze de ceux que nous avons rencontrés sont d'anciens salariés de grandes entreprises dans le secteur des industries de pointe et dans celui des services hautement qualifiés : principalement microélectronique, informatique et industrie des semi-conducteurs, aérospatiale et aéronautique, télécommunications, bureaux d'études et technocentre de l'industrie automobile, industrie nucléaire.

Ils occupaient des fonctions d'ingénieurs et de cadres (responsable de la production, directeur d'unité, directeur technique, chef de produit), ou de techniciens (techniciens de bureaux d'études, de maintenance, électroniciens) et avaient des responsabilités diverses (service formation, contrôle, 


\section{Encadré}

\section{Méthodologie de l'enquête}

L'enquête sur les entreprises installatrices d'équipements en énergies renouvelables implantées sur les communes du bassin minier de Provence constitue un des volets d'une recherche plus large portant sur ce territoire (Gallenga, Lamanthe, 2012). Cette recherche a bénéficié du soutien de l'OHM (Observatoire Hommes-Milieux) du bassin minier de Provence. Pour éviter une trop grande dispersion, nous avons choisi de centrer notre attention sur le solaire, qu'il s'agisse du solaire thermique ou photovoltaïque, sans pour autant exclure les autres domaines d'intervention des entreprises enquêtées, qu'il s'agisse des pompes à chaleur, du bois ou de la géothermie, ou d'autres activités plus traditionnelles comme la plomberie, le chauffage ou encore l'électricité. Le choix s'est porté sur les entreprises qui s'adressaient prioritairement aux particuliers, notamment pour faire écho au troisième volet du projet sur l'équipement des ménages des communes en photovoltaïque.

Plusieurs sources ont été mobilisées pour repérer les entreprises correspondant au champ de l'enquête : les sites internet des communes dont certains proposent des listes d'entreprises classées par type d'activité; le site Qualit'ENR (www.qualit-enr.org), «portail des installateurs d'énergies renouvelables », qui recense les entreprises labellisées; différents sites de professionnels du solaire; la liste des entreprises agréées par le conseil régional à l'époque où il dispensait ses propres aides; les pages jaunes de l'annuaire des Bouches-du-Rhône; des cartes de visite collectées à différentes occasions (salon des énergies renouvelables, foires, etc.).

Quarante-quatre entreprises ont ainsi pu être identifiées. Sur ce total, trente-huit entreprises ont été contactées (par courrier suivi d'un appel téléphonique) afin de proposer un entretien avec le dirigeant. Six n'ont pas pu être contactées ou ne correspondaient pas ou plus aux critères. Quatorze entretiens ont été réalisés (en face à face ou par téléphone, certains en présence de l'épouse). Sur les vingt-quatre entreprises restantes, cinq sont restées injoignables (le numéro de téléphone n'était plus attribué; nous n'avons reçu aucune réponse ou le courrier nous a été retourné); trois n'existaient plus (leur cessation d'activité a pu être vérifiée); trois ont refusé de nous accorder un entretien faute de temps et cinq parce qu'elles n'avaient plus d'activité dans le domaine des énergies renouvelables; huit ont été contactées mais il n'a pas été possible d'aboutir à un rendez-vous (difficulté du contact à se rendre disponible, multiples appels sans déboucher sur un rendez-vous); enfin, dans le cas de l'une des entreprises, le personne que nous avions contactée n'appartenait plus à la société.

L'entretien avec les dirigeants (complété par l'exploration du site internet quand il existait) avait pour objectif de recueillir des informations sur les aspects suivants : I'histoire de l'entreprise (circonstances de la création, caractéristiques du fondateur, évolutions marquantes dans le temps, orientation vers les énergies renouvelables, etc.), ses caractéristiques (taille, activité, marchés, concurrence, aire géographique d'activité, etc.), le profil du dirigeant (formation, parcours professionnel, etc.), les modalités d'insertion dans le bassin minier (raison de l'implantation dans une commune du bassin, relations locales, réseaux, collaborations, etc.), l'activité dans les énergies renouvelables (motifs et caractéristiques, positionnement, agréments, labels, insertion dans des réseaux professionnels, etc.), les caractéristiques des salariés (nombre, formation, qualification, profil et trajectoires, aires de recrutement et de résidence, etc.).

etc.) principalement dans de grandes entreprises internationales, ou des PME (petites et moyennes entreprises), bureaux d'études et cabinets de conseil, faisant partie de leurs sous-traitants et prestataires. Pour trois des dirigeants rencontrés, la création d'entreprise a accompagné l'arrivée dans le bassin minier de Provence.

Ce sont tous des diplômés du supérieur: les niveaux des diplômes obtenus s'échelonnent du BTS/DUT (brevet de technicien supérieur / diplôme universitaire de technologie) au doctorat, en passant par des diplômes de troisième cycle et des diplômes d'ingénieur ou d'écoles de commerce (voir tableau).

Ils ont créé leur entreprise dans la seconde moitié des années 2000 (plus exactement entre 2005 et 2009), au moment où les opportunités économiques dans le domaine des énergies renouvelables ont été les plus fortes, notamment grâce aux incitations fiscales et réglementaires. Ces entreprises sont de petite taille, n'emploient pour la plupart aucun salarié et ont adopté le statut d'entreprise artisanale ou bien celui de SARL (société à responsabilité limitée), quand elles ne fonctionnent pas en leur nom propre. Leur activité relève du secteur de la construction, auquel est intégrée l'installation d'équipements en énergies renouvelables. Ici, elle consiste principalement en l'installation, pour les particuliers, d'équipements en solaire thermique (chauffe-eaux solaires) ou photovoltaïque (panneaux en toiture) et de systèmes de chauffage par pompe à chaleur (PAC) ou à bois (3).

(3) Ces activités relèvent principalement du code 43 (travaux de construction spécialisés) de la NAF 2008 (nomenclature d'activités française) et, plus particulièrement, du groupe 43.2, «travaux d'installations électriques, plomberie et autres travaux d'installation", qui comprend: les travaux d'installation électrique dans tout type de locaux, les travaux de plomberie, d'installation de chauffage et de conditionnement d'air, les travaux d'installation d'eau et de gaz dans tout type de locaux et les travaux d'installation d'équipements thermiques et de climatisation. 
Tableau : Formation des dirigeants et fonctions occupées dans l'entreprise qu'ils ont quittée

\begin{tabular}{|c|c|c|}
\hline & Diplôme de formation initiale & Fonction/secteur \\
\hline \multicolumn{3}{|l|}{ «Solaristes» } \\
\hline Frédéric* & $\begin{array}{l}\text { - Diplôme d'ingénieur en mécanique } \\
\text { - DEA (diplôme d'études approfondies) génie } \\
\text { des organisations de production }\end{array}$ & $\begin{array}{l}\text { Responsable de la production et directeur adjoint de } \\
\text { la filiale d'une multinationale de la microélectronique } \\
\text { - semi-conducteurs } \\
\text { (région Paca) }\end{array}$ \\
\hline Sébastien & $\begin{array}{l}\text { - Diplômes de } 3^{\mathrm{e}} \text { cycle } \\
\text { - Doctorat en sciences de l'information }\end{array}$ & $\begin{array}{l}\text { Cadre d'une multinationale de l'informatique } \\
\text { (région Paca) }\end{array}$ \\
\hline François & Diplôme d'ingénieur & $\begin{array}{l}\text { Salarié d'une multinationale de la microélectronique (direc- } \\
\text { teur technique européen du bureau d'études) } \\
\text { (bassin minier de Provence) }\end{array}$ \\
\hline Pierre & $\begin{array}{l}\text { - Diplôme d'ingénieur } \\
- \text { Bac }+5 \text { génie physique des matériaux }\end{array}$ & $\begin{array}{l}\text { Chef de produit, consultant marketing produit dans une } \\
\text { multinationale de la microélectronique - semi-conducteurs } \\
\text { (région Paca) }\end{array}$ \\
\hline \multicolumn{3}{|c|}{ «Néo-artisans» } \\
\hline Denis & $\begin{array}{l}\text { Formation en électrotechnique et } \\
\text { électromécanique }\end{array}$ & $\begin{array}{l}\text { Postes à responsabilités (responsable de service, chef d'ate- } \\
\text { lier, responsable de la maintenance) dans différents secteurs } \\
\text { (différentes régions) }\end{array}$ \\
\hline Daniel & BTS génie climatique & $\begin{array}{l}\text { Électronicien dans l'industrie nucléaire } \\
\text { (région Paca) }\end{array}$ \\
\hline Éric & $\begin{array}{l}\text { - DUT } \\
\text { - École de commerce }\end{array}$ & $\begin{array}{l}\text { Service client, formation du personnel dans une entreprise de } \\
\text { télécommunications } \\
\text { (région Paca) }\end{array}$ \\
\hline a) Pascal & - BTS productique & $\begin{array}{l}\text { - Technocentre d'une entreprise de l'automobile } \\
\text { (région parisienne) }\end{array}$ \\
\hline b) Philippe & - Maîtrise en génie mécanique & $\begin{array}{l}\text { - Aérospatiale (bureau d'études conception assistée par } \\
\text { ordinateur) } \\
\text { (Toulouse) }\end{array}$ \\
\hline Christophe & BTS électronique & $\begin{array}{l}\text { Contrôleur aérien } \\
\text { (région Paca) }\end{array}$ \\
\hline Grégory & $\begin{array}{l}\text { - DUT } \\
\text { - BTS génie électrique, électronique, électricité }\end{array}$ & $\begin{array}{l}\text { Technicien de maintenance dans une multinationale de la } \\
\text { microélectronique } \\
\text { (bassin minier de Provence) }\end{array}$ \\
\hline
\end{tabular}

* Les prénoms sont fictifs.

\section{Ressorts et supports de la mobilité professionnelle}

Les dirigeants que nous avons rencontrés ont souhaité quitter les entreprises dans lesquelles ils travaillaient pour plusieurs raisons. Il s'agissait notamment de fuir les situations d'incertitude et d'instabilité dans lesquelles les processus continus de restructuration les plongeaient en permanence. Plusieurs d'entre eux ont ainsi connu des plans sociaux et des licenciements à répétition, des changements d'actionnaires et de stratégies, ainsi que la primauté croissante donnée aux logiques financières et de rentabilité à court terme, autant d'éléments qui enlèvent de la visibilité sur l'avenir, tant en termes d'emploi que sur l'évolution des salariés au sein de l'entreprise. La concurrence internationale, particulièrement aiguë dans le domaine des hautes technologies, a visiblement contribué à accentuer ces incertitudes. Pour d'autres, l'objectif était de s'extraire de conditions, relations ou ambiances de travail dégradées (pressions, recherche de la rentabilité à tous crins, mauvaises relations au sein de l'entreprise, cas de suicides au travail, etc.), à propos desquelles ils expriment leur insatisfaction et leur saturation ( ras-le-bol», " marre de la grosse société», "refus de travailler dans une grande entreprise », etc.). La création d'entreprise répond, a contrario, à leur aspiration à une plus grande indépendance ("ne plus avoir à rendre des comptes», "éviter la routine») qui explique leur refus de chercher un nouvel emploi dans une entreprise similaire.

Les plans sociaux, licenciements économiques ou dispositifs d'essaimage (4), qui offrent des ressources financières - indemnités, maintien du salaire, possibilité de réintégration -, constituent à la fois un filet de sécurité et un soutien aux investissements dans la nouvelle activité. Ils sont saisis comme des opportunités pour partir et faire autre chose. Les dirigeants que nous avons rencontrés ont ainsi expérimenté l'ensemble des voies de sortie disponibles, y compris la démission. Lors de leur mobilité, ils cherchent à concilier un désir d'autonomie et de

(4) L'essaimage est un dispositif mis en place par les grandes entreprises pour aider certains de leurs salariés à créer ou à reprendre une entreprise. Cette aide peut prendre plusieurs formes: soutien financier (prêt, subvention, maintien du salaire), aide aux démarches, formation, relations économiques (client-fournisseur), possibilité de réintégration, etc. Il s'intègre dans des stratégies de gestion des «sureffectifs» et/ou de rentabilité économique (relations de sous-traitance de l'entreprise créée avec l'entreprise d'origine, développement de marchés de niche, de technologies spécifiques, production de petits volumes, etc.). 
sécurité - par la réalisation d'un projet personnel ou familial qui va leur permettre de s'assurer un revenu - et l'aspiration à une nouvelle qualité de vie et de travail : la grande entreprise n'apparaît plus en mesure de leur garantir tout cela, si bien que ce sont les petites structures qu'ils projettent de créer qui portent désormais leurs aspirations. On rejoint ici des observations faites par ailleurs (Росніс, 2001), qui montrent que les cadres reportent les attributs classiques de leur emploi passé en entreprise ${ }^{(5)}$ sur leurs attentes liées à la création d'entreprise. C'est pourquoi, lors des entretiens, ces dirigeants n'ont pas exprimé de sentiment de déclassement par rapport à leur position professionnelle antérieure. Même si certains nous ont indiqué que leurs revenus actuels sont inférieurs à ceux qu'ils avaient auparavant, les gains en termes de conditions de vie et de travail, l'amélioration de la visibilité sur leur avenir, la pratique d'une activité socialement et symboliquement valorisée (écologie, énergies renouvelables, travail indépendant, etc.) développée à leur initiative et non par défaut, semblent, à tout le moins dans leurs discours, compenser les pertes pécuniaires.

\section{Sortir de l'expérience des licenciements à répétition et des logiques financières}

Créer son entreprise est, pour François, la voie qu'il a privilégiée pour sortir de la difficile expérience des licenciements à répétition qu'il a connus en tant que directeur technique du bureau d'étude d'une multinationale de la microélectronique. Il a en effet été licencié une première fois en 2003 puis, après une succession de rachats, il l'a été à nouveau en 2008. La création d'une entreprise, nous dit-il, c'est aussi s'assurer d'une meilleure projection dans le temps, qui n'est plus permise par la situation de perpétuelle instabilité et d'incertitude inhérente à l'entreprise multinationale dans laquelle il était. De la même manière, le refus des logiques financières dictées par les actionnaires et la crainte de connaître le chômage sont les motivations exprimées par Pierre pour lancer sa société. Salarié d'un groupe de semi-conducteurs, il quitte son emploi dans le cadre d'un plan de départs volontaires en 2002. Il rejoint un autre groupe, cette fois dans l'instrumentation, qui finit par être racheté; or ce que lui proposent les nouveaux actionnaires, explique-t-il, ne lui convient pas. Par ailleurs, il pense que l'avenir des hautes technologies se situe désormais davantage dans les pays émergents qu'en Europe, ce qui lui fait craindre de se retrouver, à terme, au chômage. Par deux fois,

(5) Les attributs classiques de l'emploi cadre sont aux antipodes des caractéristiques désormais adoptées par les modalités de gestion des ressources humaines privilégiées par les entreprises multinationales (incertitude, instabilité et mobilité, notamment, qui apparaissent ici comme des repoussoirs). il quitte ainsi son emploi dans des conditions financières qu'il présente comme intéressantes :

«Je savais que la haute technologie, c'était mort, et qu'avec tous les Indiens, Roumains ou Russes qu'on forme, l'ingénieur, il a plus vraiment sa place, ou, enfin, il l'aura plus dans vingt ans. Moi, j'ai 40 ans, je vais bosser jusqu'à 65 voire 70 peut-être; donc, si c'est pour me retrouver au chômage comme mes aînés dans cinq ou dix ans, ça m'intéressait pas. Deuxièmement, à deux reprises, j'ai dî quitter mon entreprise parce qu'on m'en a offert la possibilité dans des conditions financières intéressantes; donc, j'en ai profité, j'ai utilisé cet argent pour construire une extension chez moi, que je loue. Je l'ai construite dans deux buts : parce que c'était ma passion, le bricolage; et parce que, c'était, je pense, financièrement là où je pouvais mettre mon argent le plus sûrement, c'est-à-dire que derrière je pouvais louer, ça me rapportait une rente.»

\section{"Quitter un truc que je ne supportais plus"}

Éric quitte l'entreprise de télécommunications où il travaille au service client et à la formation du personnel après avoir décidé de bénéficier d'un dispositif d'essaimage dont l'objectif était d'inciter au départ certaines catégories de salariés, principalement les fonctionnaires recrutés du temps où l'entreprise était publique. Lui-même n'est pas directement visé car il a été embauché en contrat à durée indéterminée (CDI) de droit privé. Le dispositif lui permet de percevoir son salaire pendant un an avec la possibilité de réintégrer l'entreprise pendant cinq ans. Par ailleurs, il a droit à un congé individuel de formation financé par le Fongecif. Mais, d'après lui, c'est surtout le climat qui régnait au sein de l'entreprise - notamment suite à son entrée dans le secteur privé qui a conduit agents de la fonction publique et salariés de droit privé à se côtoyer - qui a motivé son souhait de partir :

«Il y avait une porte ouverte qui s'appelait essaimage; donc j'ai fait déjà un Fongecif, je me suis adressé au Fongecif, ensuite je me suis lancé. [...] Au départ c'était pour les fonctionnaires et pas pour les contractuels; c'était autorisé pour les contractuels mais l'objectif, c'était de dégraisser. En fait, c'est pas moi qui devais partir. [...] Je ne quittais pas un truc que je ne supportais plus pour me retrouver salarié d'une autre boîte, ça n'avait aucun intérêt. Donc l'idée c'était de me lancer...»

\section{Servir des projets familiaux et une meilleure qualité de vie}

Pascal et Philippe sont deux amis qui se sont rencontrés dans leur ancien emploi. Le premier, Pascal, travaillait dans le technocentre d'une grande entreprise de l'industrie automobile, en région parisienne. Il démissionne pour rejoindre Philippe, déjà installé en Provence, dont la société de géothermie récemment créée lui assure un emploi ( De toute façon, il y avait du taf»). Philippe, quant à lui, travaillait dans un bureau d'études lié à une société 
d'aéronautique et prestataire du technocentre. Il démissionne pour suivre son épouse infirmière, qui a obtenu une mutation dans le Sud. Ce motif lui permet de bénéficier des allocations chômage et d'une formation. Pascal évoque pour sa part des arguments de qualité de vie et le projet de construire une maison, projet qui était inaccessible en région parisienne :

«J'ai débarqué ici, parce que dix ans de Paris, dix ans de technocentre, je saturais; donc je voulais voir un autre volet [...], j'en avais marre. On avait le projet de construire, mais dans la région parisienne, ce n'est pas possible ou trop loin du lieu de travail; il fallait faire des kilomètres et des kilomètres pour faire construire une petite maison [...]. Ras le bol de ces grosses entreprises et essayer autre chose; donc on a fait bingo, on a dit: "Allez on y va"; avec ma femme, on était à $200 \%$ pour partir; bon on essaie, on verra.»

Sa femme, alors employée en région parisienne chez un loueur automobile, pose sa démission en même temps que lui.

Ce sont aussi des arguments d'ordre familial et de qualité de vie qu'évoque Daniel qui, dans son emploi précédent, était amené à travailler neuf mois sur douze à l'étranger: "Ma femme m'a dit: "Je n'ai pas épousé un fantôme." Alors j'ai décidé de rester sur place. "

Plus largement, chez tous les entrepreneurs rencontrés, la mobilité professionnelle s'articule à un ancrage territorial particulièrement marqué. Il concerne aussi bien ceux qui se sont récemment installés sur le territoire (et pour lesquels mobilité professionnelle et mobilité géographique se confondent) que ceux qui, anciens salariés d'entreprises implantées localement, résidaient déjà sur le territoire. En couple, ces entrepreneurs souhaitent accéder à la propriété individuelle, ce qui a une incidence sur les choix qu'ils opèrent dans le cadre de leurs projets professionnels (volonté de rester sur le territoire). Il est d'ailleurs remarquable que, à la question "Pourquoi avez-vous installé votre entreprise sur une commune du bassin minier?», la plupart répondent que c'est parce qu'ils y habitent (la résidence familiale est généralement le siège de l'entreprise, notamment parce qu'ils n'ont pas les moyens de louer un local professionnel). Ces projets professionnels ont ainsi une forte dimension familiale : soit que la mobilité s'inscrive dans un projet familial explicite comme dans l'exemple de Pascal et de Philippe ci-dessus, où les deux conjointes sont concernées par la mobilité; soit qu'elles aient été associées à la décision et la soutiennent en assurant, par leur emploi, une rentrée financière régulière (c'est le cas pour Éric dont l'épouse est employée de banque) et/ou en prenant en charge les tâches de gestion (l'épouse de Grégory, employée dans une entreprise locale d'informatique, assure le travail administratif). Dans un seul cas toutefois, celui de Denis, l'épouse est associée et travaille avec lui dans l'entreprise. Comme d'autres travaux l'ont montré, le rôle du conjoint, ici celui de l'épouse, est primordial au moment où est prise la décision d'installation: son aval est déterminant en même temps que sa position dans le ménage, inactive ou salariée, conditionne le type de soutien qu'elle sera en mesure d'apporter ${ }^{(6)}$.

\section{Les énergies renouvelables comme opportunité}

Le développement du marché des énergies renouvelables est l'autre opportunité sur laquelle ces «néo-entrepreneurs» se sont appuyés pour servir leur projet de mobilité. Il leur a permis de combiner rentabilité économique, régularité de revenu et aspiration à gagner en autonomie à travers la création d'une entreprise. Ainsi, Sébastien déclare avoir réalisé, en 2005, une étude d'opportunité qui lui a révélé "que tous les voyants étaient au vert concernant le photovoltaïque», ce qui l'a décidé à lancer son entreprise. Pierre remarque que «dans le bâtiment, on a toujours besoin de monde» et que, du fait des nouvelles réglementations, comme "trente et un millions de maisons sont à reprendre en France, il y a du travail pour trente ans ». Il avait par ailleurs testé et confirmé son envie en construisant sa propre maison. Éric, quant à lui, souhaitait s'installer comme artisan. En mettant en regard les études qu'il avait faites, sa formation et le marché ( «dans le bâtiment, il y a du boulot»), il s'est rendu compte que deux possibilités s'offraient à lui : électricité et plomberie. N'étant pas bon en électricité, dit-il, il a opté pour la plomberie.

Nouveaux venus dans ces activités, afin de pouvoir les exercer, ils ont dû acquérir des compétences qu'ils ne possédaient pas. Dans ce domaine aussi, les dispositifs de sortie des grandes entreprises ont servi de tremplin en leur apportant les ressources nécessaires à travers, notamment, les possibilités de formation et les aides à la reconversion qui leur sont associées.

\section{Des ex-salariés en reconversion}

Pour les anciens salariés rencontrés, il a fallu acquérir les compétences techniques des métiers concernés : sur les énergies renouvelables, mais aussi plus largement sur la construction de bâtiment, où de nombreuses connaissances sont nécessaires pour les travaux de plomberie, chauffage, couverture, etc. Parallèlement, ils ont dû se former à tout ce qui relève de la conduite d'une entreprise: gestion et cadre réglementaire liés à l'inscription au registre des métiers pour les entreprises artisanales, inscription pour laquelle est exigée la détention d'un diplôme professionnel attestant la qualification de l'artisan dans le métier exercé.

(6) Voir, par exemple, Testenoire, 2000; Pochic, 2001. 
Les «néo-entrepreneurs» ont largement mobilisé les dispositifs publics qui ont encadré leur départ de leur entreprise en restructuration. Plusieurs d'entre eux, en effet, ont bénéficié de formations dans le cadre de dispositifs de reconversion en tant que demandeurs d'emploi ou bien via les dispositifs d'essaimage (congé individuel de formation). Trois d'entre eux ont obtenu des diplômes de l'enseignement supérieur par cette voie, dans des spécialités orientées vers la gestion de l'entreprise : DESS (diplôme d'études supérieures spécialisées) finance, DESS communication et licence de gestion. Trois autres ont suivi des formations conduisant à des certifications professionnelles leur permettant de s'inscrire au registre des métiers. C'est le cas de Pierre, qui a passé un CAP d'installateur en solaire thermique grâce à une formation récemment mise en place par le Greta ${ }^{(7)}$ d'un lycée d'une commune voisine dans le cadre du plan régional de formation professionnelle financé par le conseil régional et ouvert aux demandeurs d'emploi. De même, Éric a décroché un CAP de plombier chauffagiste et Pascal a passé un baccalauréat professionnel en génie climatique à l'Afpa (Association nationale pour la formation professionnelle des adultes).

Ainsi, phénomène relativement inédit en France, des salariés hautement qualifiés et titulaires de diplômes de l'enseignement supérieur sont amenés à préparer des diplômes des premiers niveaux de l'enseignement professionnel. Pour autant, leur formation initiale, tout comme l'expérience professionnelle qu'ils ont acquise dans les grandes entreprises dans lesquelles ils ont exercé ne sont pas sans utilité dans leur nouvelle activité. Leurs connaissances sont mobilisées de différentes façons, qu'ils s'agissent des compétences techniques acquises lors de leur formation initiale (électrotechnique, électronique, génie climatique, génie électrique) qui sont réutilisées dans le domaine des énergies renouvelables, ou de leurs compétences en informatique particulièrement utiles pour la gestion des stocks et la création de sites internet ou encore, de leurs capacités à se documenter et à monter les dossiers permettant à leurs clients d'accéder aux aides à l'installation.

En outre, ils ont dû acquérir les certifications Qualit'ENR nécessaires pour que leurs clients puissent bénéficier des aides publiques. En effet, les aides des collectivités locales ne peuvent être versées que si les clients font appel à des professionnels possédant cette reconnaissance, gage officiel de

(7) «Les Greta sont les structures de l'éducation nationale qui organisent des formations pour adultes dans la plupart des métiers. On peut aussi bien y préparer un diplôme du CAP au BTS que suivre un simple module de formation.» (Source : http://www.education.gouv.fr/cid50753/la-formation-continue-des-adultes-a-l-education-nationale.html; consultée le 30 juin 2014.) leur compétence, également fortement recommandée pour les aides nationales.

Tous les enquêtés insistent sur le rôle important joué par les fournisseurs de matériel dans l'acquisition des connaissances techniques. La formation sur le tas, évoquée par la plupart des dirigeants rencontrés, est une autre voie, souvent complémentaire. Pour plusieurs d'entre eux, et particulièrement quand ces dirigeants font partie de ménages accédant à la propriété individuelle, la construction de leur maison leur a donné l'occasion d'acquérir différentes techniques (couverture, charpente, électricité, plomberie, etc.); l'installation à leur domicile de différents équipements en énergies renouvelables leur a permis de tester le matériel et leurs propres compétences; enfin, ils ont pu évaluer techniques et produits par les diverses activités de bricolage auxquelles ils se sont livrés. Les expériences professionnelles ponctuelles qu'ils ont pu faire après avoir quitté leurs entreprises initiales, en tant que salariés ou en tant que stagiaires, ont aussi été des occasions de se former: Denis a été employé comme commercial dans une entreprise d'installation en énergie solaire, si bien qu'il a pu participer à différents chantiers en compagnie du plombier de l'entreprise; Daniel a travaillé avec un ami à la pose de cuisines et effectuait les travaux de plomberie; Pascal a appris sur le tas, dans la société de géothermie créée par Philippe; avant de créer sa propre société, Grégory a été employé pendant quelque temps dans une entreprise d'installation de panneaux photovoltaïques.

\section{Des mobilités au carrefour d'un ensemble de facteurs}

Au-delà de ces cas individuels, certes peu nombreux mais concentrés du point de vue de l'implantation géographique et des trajectoires socioprofessionnelles, des tendances plus générales dont ils sont, pour partie, le produit et le révélateur, semblent à l'œuvre. Les trajectoires de sortie de la grande entreprise débouchant sur la création de petites structures sont rendues possibles et prennent leur sens en raison de l'articulation entre un ensemble de facteurs. D'une part, les transformations des systèmes productifs et d'emploi - s'y conjuguent les stratégies des grandes entreprises et les orientations des politiques publiques en la matière - se combinent ici avec la montée des préoccupations environnementales dans la société et les mesures de soutien à la «transition énergétique», qui ont ouvert de nouvelles opportunités de marché. D'autre part, dans un contexte de chômage de masse et de recomposition du salariat, les conditions sont propices au réinvestissement du travail indépendant, notamment sous la forme de l'entreprise artisanale. 


\section{Restructurations, incitations au départ volontaire et réinvestissement du travail indépendant}

Si la dégradation des conditions de travail et d'emploi associée aux restructurations en continu conduit certains salariés sur la voie de l' «exit», cette décision trouve un écho particulier dans les dispositifs mis en place par les directions pour se séparer des salariés et gérer les sureffectifs.

En plus des plans sociaux engageant des licenciements économiques collectifs, les procédures mises en œuvre sont de plus en plus variées et individualisées avec, notamment, la possibilité de «rupture conventionnelle» autorisée par la loi $\mathrm{n}^{\circ}$ 2008-596 «portant modernisation du marché du travail» ou encore l'appel aux départs volontaires dans le cadre des «plans de sauvegarde de l'emploi» (nouvelle appellation des "plans sociaux») qui prévoient la réduction des effectifs (Kerbourc' $\mathrm{H}, 2007$; BeAujolin-Bellet et al., 2012). La «rupture conventionnelle» est une rupture du contrat de travail qui se fait d'un commun accord entre le salarié et l'employeur, et qui permet au salarié de percevoir les allocations chômage, contrairement à la démission. De la même façon, dans les dispositifs de départs volontaires, qui se sont fortement développés dans les années récentes (BEAUJOLIN-BELlet et al., 2012), l'accès aux droits et indemnités attachés au licenciement pour motif économique est assuré : indemnités de départ et allocations de chômage, accompagnement à la reconversion (formation, aide à la création d'entreprise), etc.

Des travaux ont montré que le «succès» rencontré par les dispositifs de départs volontaires tient au fait que cette procédure combine tout à la fois les visées des entreprises et l'aspiration de certains salariés, dès lors que ceux-ci peuvent partir en ayant accès à certaines garanties, notamment sur le plan financier. Les départs volontaires n'ont certes pas la même résonance ni les mêmes conséquences pour les salariés selon leur niveau de qualification, leur âge ou leur secteur d'activité. Ils permettent cependant à des motivations individuelles exprimées par nombre d'entre eux de se concrétiser, ce qui laisse penser qu'ils peuvent être vécus comme une occasion de fuir l'entreprise et le mal-être au travail et/ ou comme une opportunité de réaliser un projet professionnel de reconversion tout en profitant des mesures d'accompagnement et des indemnités prévues à cet effet (BOURGUIGNON, GARAUDEL, 2012).

La création d'entreprise et, plus largement, la sortie du salariat vers le travail indépendant, peut constituer une voie de reconversion pour ces ex-salariés. Elle trouve un écho particulièrement favorable dans les périodes de chômage et/ou de dégradation des conditions d'emploi. Elle a par ailleurs bénéficié, depuis les années 1980, à la fois du regain d'intérêt accordé, dans les représentations sociales, à l'entreprise et à l'entrepreneuriat et d'incitations nouvelles dans le cadre de la politique publique de l'emploi. En effet, à partir de la fin des années 1970, la puissance publique a considéré la création d'entreprise comme étant à même de servir un double objectif: la redynamisation $\mathrm{du}$ tissu productif (notamment à travers le soutien aux petites entreprises) afin de favoriser la création d'emplois et, partant, la lutte contre le chômage. Des dispositifs de différents types ont été mis en place pour faciliter la création d'entreprise, via le soutien financier et les incitations fiscales, la facilitation des démarches, la définition de nouveaux cadres juridiques ainsi que l'accompagnement des porteurs de projet (plateformes d'initiatives locales, couveuses d'entreprises, etc.). Les demandeurs d'emploi ont été une cible privilégiée de ces dispositifs, dans une histoire qui compte désormais plusieurs décennies comme le montre Fanny Darbus (2008). À la fin des années 1970, avec l'installation du chômage de masse, le lancement de l'Accre (aide aux demandeurs d'emploi créant ou reprenant une entreprise, ouverte aux demandeurs d'emploi susceptibles d'être indemnisés) témoigne d'une volonté de «promotion institutionnelle de l'auto-emploi». Par la suite, dans les années 2000, il s'agit de mettre sur pied des dispositifs dont l'objectif est la «sécurisation» et l'aménagement des transitions professionnelles, du chômage vers l'entrepreneuriat. Pour cela, les aides sont accordées aux demandeurs d'emploi porteurs de projet à condition qu'ils aient recours aux services d'accompagnement; dans le même temps, ils ont la possibilité de cumuler les revenus de leur nouvelle activité avec les minima sociaux ou les indemnités chômage. Dans ce sens, le Cape (contrat d'appui au projet d'entreprise) instauré en 2003 par la loi «Dutreil» vise à faciliter la transition vers l'entrepreneuriat à travers des aménagements administratifs, juridiques et fiscaux (selon les cas, maintien de l'affiliation au régime de protection sociale des salariés; maintien de la perception des revenus de transferts sociaux) (DARBUS, 2008).

Le renouvellement de l'artisanat est en quelque sorte une conséquence et un révélateur de cette conjonction entre projets individuels de reconversion et incitations, institutionnelle et symbolique, à la création d'entreprise. Depuis les années 1980, l'artisanat est en effet traversé par de nouvelles dynamiques. D'une part, il a lui-même fait l'objet d'aménagements administratifs, juridiques et fiscaux visant à faciliter les installations. De l'autre, il a été investi par de nouveaux profils de travailleurs. Comme le fait ressortir Caroline MAZAud (2012), alors qu'il constituait traditionnellement une filière de promotion ouvrière, il voit aujourd'hui son recrutement s'élargir et les profils de ses dirigeants se diversifier dans la mesure où il représente désormais «un filet de sécurité pour des reconvertis issus des classes moyennes » et attire des populations plus diplômées, par exemple d'ex-cadres de l'industrie ou des services, en reconversion 
professionnelle, ayant quitté leur emploi à l'occasion d'un plan social. Pour l'auteure, les mobilités initiées par ces nouveaux entrants témoignent tout à la fois d'un «désenchantement» par rapport au contexte dans lequel ils ont évolué jusqu'alors (la grande entreprise entre autres), des dégradations subies par la condition salariale (incertitude, crainte du chômage) et d'un désir de changement de leurs conditions d'emploi et de travail.

Ainsi, l'artisanat est devenu attractif pour des personnes en quête de mobilité, qu'il s'agisse d'anciens cadres d'entreprises en rupture avec leur vie professionnelle précédente ou de salariés en cours de carrière qui s'engagent dans un second métier (Legros, 2009) ${ }^{\left({ }^{(8)}\right.}$. Ils sont motivés tout à la fois par un désir d'indépendance et celui de ne plus être soumis à la hiérarchie; ils souhaitent également échapper au stress des objectifs à atteindre et aux critères de rentabilité «sans foi ni loi», en même temps que vivre une «passion» ou se lancer dans une «aventure », même si la réalité qu'ils rencontrent une fois installés ne correspond que très partiellement à leurs attentes. Dans le secteur de la construction, les énergies renouvelables ont largement contribué à ce mouvement. Elles ont été à l'origine d'une modification de la sociologie des professions et métiers concernés. Les installateurs/mainteneurs, assembleurs et techniciens en énergies renouvelables sont en partie venus supplanter les métiers traditionnels de plombier/chauffagiste, électricien, frigoriste, couvreur, etc. (Colombard-Proust et al., 2007). Attirés par le travail indépendant et les énergies renouvelables qui bénéficient d'une représentation valorisée et valorisante, de nouveaux profils d'entrepreneurs ont investi le secteur.

\section{La transition énergétique : opportunités économiques, politiques publiques et dynamiques entrepreneuriales}

Dans les trajectoires de mobilité étudiées ici, les impacts des transformations des tissus productifs, des stratégies des entreprises et des modes de gestion de l'emploi ont «rencontré» les opportunités économiques ouvertes par la montée des préoccupations environnementales dans la société et la volonté politique d'organiser la «transition énergétique». Ces dernières ont en effet contribué à l'émergence de nouveaux marchés et de nouvelles activités, comme l'installation d'équipements en énergies renouvelables, dont se sont emparés les

(8) Selon une étude réalisée en 2007-2008 par l'Institut supérieur des métiers, seule la moitié des artisans des secteurs de la coiffure, du bâtiment et de l'alimentation détient le premier niveau de diplôme professionnel pour exercer une activité artisanale (CAP [certificat d'aptitude professionnelle] ou BEP [brevet d'études professionnelles]) tandis que $20 \%$ d'entre eux sont diplômés de l'enseignement supérieur (étude rapportée par Legros, 2009). «néo-entrepreneurs» de notre échantillon pour réaliser leur projet de création d'entreprise.

Ces perspectives économiques s'expliquent largement par les politiques publiques mises en place en matière environnementale à partir de la seconde moitié des années 2000. L'État a joué un rôle prépondérant de trois manières : en incitant (création d'une demande); en soutenant financièrement (solvabilisation de la demande); et en réglementant (prescriptions normatives, orientation des activités). C'est avec le lancement du «Grenelle de l'environnement» à la fin de l'année 2007 que cette politique prend toute son ampleur. Cette consultation a débouché sur un ensemble législatif aux implications directes en matière économique, qui s'articule autour de mesures visant à une plus grande maîtrise de la consommation énergétique et à la réduction du recours aux énergies fossiles au profit des énergies renouvelables; la loi dite "Grenelle $1 »$ (votée en 2009) établit une série d'objectifs à atteindre d'ici 2020. Avec la «croissance verte» (nouvelles activités et «verdissement» des activités existantes) et le besoin de réponses aux problématiques environnementales (traitement des déchets, dépollution, etc.), la transition énergétique apparaît comme un gisement pour la création d'activité et d'emplois dans l'avenir, particulièrement dans le secteur de la construction, directement impliqué par ces nouvelles politiques ${ }^{(9)}$.

La réduction attendue des dépenses énergétiques implique en effet la rénovation thermique des bâtiments et de nouvelles normes et réglementations pour les constructions neuves (réglementation thermique, performance énergétique, bâtiments basse consommation, etc.) ou encore, le développement du recours aux énergies renouvelables. Des mesures financières incitatives en direction des particuliers accompagnent ces prescriptions : l'éco-prêt à taux zéro dont le but est de favoriser la réalisation d'un ensemble de travaux en vue d'améliorer la performance énergétique des habitations (comme, par exemple, l'isolation des murs et l'équipement en chauffe-eau solaire); le crédit d'impôt pour l'installation d'équipements en énergies renouvelables (solaire, bois, pompes à chaleur, etc.), pour les travaux d'isolation, etc.; la TVA (taxe sur la valeur ajoutée) à 5,5\% pour ce type de travaux; etc. La production directe d'énergie par les particuliers est fortement encouragée, notamment par la subvention à l'installation de panneaux photovoltaïques en toiture, la revente d'électricité à EDF se faisant selon un tarif incitatif. Ces dispositifs sont cumulables et, selon les pouvoirs publics, peuvent dans certains cas permettre de subventionner les travaux

(9) Dans son rapport de 2010, le Conseil d'orientation pour l'emploi prévoyait la création de 600000 emplois en relation avec la «croissance verte», dont la moitié devait se situer dans le secteur de la construction (CONSEIL D'ORIENTATION POUR L'EMPLOI, 2010). 
à hauteur de $50 \%$ de leur coût. Certaines collectivités locales complètent les aides de l'État et viennent abonder la subvention de certains équipements (l'installation de chauffe-eau solaire par exemple).

L'incitation au recours à des professionnels labellisés traduit la volonté des pouvoirs publics de réguler, un tant soi peu, l'entrée d'entreprises sur un marché qui, au moment où ces mesures ont été mises en place, a attiré de nombreux candidats et a connu des problèmes de qualité récurrents ${ }^{(10)}$. De même, le moratoire de décembre 2010 sur la production d'électricité photovoltaïque (en direction cependant des seuls bâtiments professionnels et édifices publics, les particuliers n'étant pas concernés) et l'instauration d'un nouveau cadre de régulation (système d'appels d'offres et d'autorisation, réduction du tarif de revente) témoignent des efforts de la puissance publique pour mettre de l'ordre dans cette activité émergente à la croissance jugée anarchique. Enfin, les conditions d'obtention des aides aux particuliers se sont durcies et ont fait l'objet de nouvelles clauses (performance et types d'équipements), tandis que dans le contexte récent de la crise, certaines collectivités locales ont décidé de supprimer leurs subventions. Ainsi, le marché qui était apparu comme particulièrement porteur et avait suscité l'intérêt de nombreuses entreprises a vu les conditions d'exercice des entreprises du domaine rapidement modifiées. Certaines se sont donc retirées (c'est notamment le cas d'artisans du bâtiment qui ont tenté de compléter leur offre en se lançant dans l'installation d'équipements en énergies renouvelables). D'autres, spécialement créées pour cette activité, ont disparu, comme nous le verrons plus bas.

C'est dans ce cadre général, où le rôle de l'État apparaît structurant tant par sa capacité à faire apparaître de nouveaux débouchés économiques qu'à définir les conditions d'exercice des activités ainsi créées et les conduites économiques compétitives (Fligstein, 2005; Bourdieu, 2000), que se déploie l'activité des néo-entrepreneurs, entre opportunités, réglementation et régulations, ouverture et réduction des aides, fluctuations des politiques publiques. C'est aussi au niveau des territoires dans lesquels ils sont installés que ces mobilités prennent leur sens, et particulièrement dans les dynamiques territoriales confrontées à des reconfigurations productives, politico-administratives et sociodémographiques, qui

(10) Les perspectives de rentabilité, notamment dans le domaine du photovoltaïque, paraissaient particulièrement prometteuses grâce aux diverses incitations mises en place. De nombreuses sociétés, dont l'activité première était la vente d'équipements et qui ne possédaient pas les compétences techniques en matière d'installation, se sont donc lancées. Elles ont démarché les particuliers et vendu de nombreuses installations en toiture qui n'ont pas atteint le niveau de rentabilité escompté au regard des investissements occasionnés en raison de malfaçons ou de mauvaise qualité de l'installation (orientation au nord, fuites dans les toits, etc.). constituent tout à la fois un contexte de contraintes, d'opportunités et de ressources.

\section{Dynamiques territoriales et ressources locales}

Le bassin minier de Provence possède une histoire industrielle liée à l'énergie : pendant plus d'un siècle, l'exploitation de mines de lignite y alimentait, notamment, une centrale thermique. Son exploitation est définitivement arrêtée en 2003, mais elle a été anticipée de longue date par Charbonnages de France, gestionnaire du site, à travers un ensemble d'incitations à l'implantation de nouvelles entreprises dès la fin des années 1970 puis, par les pouvoirs publics, qui ont mis en œuvre une stratégie explicite de reconversion à partir du milieu des années 1980. Ces efforts, en particulier via la création de zones d'activité et le déploiement $\mathrm{du}$ fonds d'industrialisation des bassins miniers, ont été guidés par une volonté de réindustrialisation du territoire. Ils ont favorisé l'émergence d'un tissu industriel de haute technologie avec l'installation fortement soutenue par des aides publiques (Garnier, Lanciano, 2004) d'établissements de multinationales de la microélectronique dans une de ces zones. La transition productive du territoire, d'activités organisées autour de l'industrie lourde (extraction minière, transformation du minerai, production d'électricité) aux nouveaux secteurs industriels de haute technologie, a ainsi bien eu lieu. Cette reconversion a pour conséquence non seulement l'évolution de la composition sectorielle des activités mais aussi celle de la population active ayant un emploi dans les communes du bassin, dont la vocation résidentielle devient plus marquée (accession à la propriété en habitat pavillonnaire) ${ }^{(11)}$.

Les entreprises dans le domaine de l'installation d'équipements en énergies renouvelables ont pour partie été créées, comme nous l'avons dit, par d'anciens cadres, ingénieurs et techniciens, auparavant salariés des grandes entreprises de la microélectronique implantées sur le bassin mais aussi dans les territoires voisins, comme celui d'Aubagne-La Ciotat. Celui-ci a lui-même été l'objet d'une reconversion suite à la fermeture des chantiers navals et a bénéficié de fonds qui ont permis l'implantation de nouvelles zones d'activité (GARNIER et al., 2004). Ainsi, les mobilités des néo-entrepreneurs s'inscrivent dans le cadre des reconfigurations

(11) Selon le recensement général de la population, l'industrie et le BTP (bâtiment et travaux publics) regroupaient 50\% de l'emploi total en 1982 , pour $38 \%$ en 1990 . En 2008, près de $70 \%$ de la population active travaillant dans le bassin est employée dans des activités tertiaires, pour $29 \%$ en 1968 , et $32 \%$ dans l'industrie et le BTP, pour $64 \%$ en 1968 . La part des ouvriers s'élève à $21 \%$ en 2008 , pour $60 \%$ en 1968 ; c'est en 1999 que cette catégorie perd le premier rang au profit des professions intermédiaires. Les cadres et professions intellectuelles supérieures ont connu la plus forte progression, passant de $3 \%$ en 1968 à près de $19 \%$ en 2008 . 
productives protéiformes de ces territoires: à la suite de la reconversion du bassin, qui se traduit par l'implantation de nouvelles activités venues compenser la fermeture des anciennes industries; aussi, ensuite, au travers des processus continus de restructuration caractérisant les nouvelles activités. Certains des «néo-entrepreneurs» rencontrés ont, quant à eux, choisi de lancer leur entreprise sur le bassin minier de Provence car la vocation résidentielle du territoire, elle-même associée aux reconfigurations productives, leur permet de trouver des débouchés pour leur activité.

Sur le plan territorial, les mobilités observées ont lieu sur l'ensemble de l'aire urbaine de MarseilleAix-en-Provence, qui fournit un ensemble de ressources. Avec 1,6 million d'habitants et plus de quatre-vingts communes autour des deux grandes villes, se développent en périphérie des centres urbains de vastes zones à vocation résidentielle dédiées à l'habitat pavillonnaire. Ces zones de déconcentration urbaine, qui connaissent une croissance rapide, offrent un vaste marché pour les spécialistes de la construction, notamment dans les énergies renouvelables : les particuliers y sont nombreux à demander l'implantation de panneaux photovoltaïques en toiture et/ou l'installation de chauffage et chauffe-eau fonctionnant avec des énergies vertes.

Le conseil régional a mis en place un ensemble de mesures et de dispositifs s'adressant aux entreprises dont l'objectif est de structurer une filière à même de répondre à la demande, en particulier dans le secteur de la construction. Ainsi, le Pôle régional d'innovation et de développement économique solidaire (Pridès) dédié au bâtiment durable en Méditerranée, créé en 2008, se présente comme «un regroupement interprofessionnel d'acteurs du bâtiment engagés sur la voie du développement durable ${ }^{(12)} \gg$ regroupant plus de deux cents adhérents (maîtres d'ouvrage et maîtres d'œuvre, entreprises et artisans, organismes de formation, organisations professionnelles, syndicats, chambres consulaires, centres de ressources). Sa mission consiste à développer les activités et la compétitivité des entreprises régionales de la construction «en les accompagnant dans la mutation de leurs pratiques vers l'action durable, par la montée en qualité et en quantité des opérations bâtiments durables méditerranéens, à travers un réseau interprofessionnel ${ }^{(13)} \gg$. D'autres dispositifs poursuivent des objectifs similaires en direction des entreprises de la construction : accompagnement technique et financier des projets de lutte contre le changement climatique et d'amélioration de la gestion de l'énergie; divers soutiens à

(12) http://www.b2match.eu/batimentdurable2014/participants/62; consulté le 2 juillet 2014 .

(13) Pour en savoir plus sur l'association interprofessionnelle «Bâtiments durables méditerranéens», voir le site : http:// polebdm.eu/; consulté le 2 juillet 2014. la filière pour favoriser la création d'emplois grâce aux activités de rénovation thermique ${ }^{(14)}$. Dans le même sens, des actions sont mises en œuvre pour inciter les petites entreprises à se regrouper afin d'être en mesure de proposer une offre globale. Une formation d' «installateur mainteneur en systèmes solaires et photovoltaïques», ouverte aux demandeurs d'emploi, a été mise en place de 2006 à 2008 dans le cadre du plan régional de formation et a accueilli une quinzaine de stagiaires par promotion. Cette initiative faisait suite au constat de divers acteurs locaux (élus, mission locale, professionnels, Greta, etc.) d'une carence en compétences et en offre locale de formation pour faire face à la montée des besoins en main-d'œuvre suscités par la croissance de l'activité.

Tous ces éléments ont joué dans la dynamique de création de petites unités installatrices d'équipements en énergies renouvelables sur ce territoire. Ils ont en effet constitué le creuset de ressources et opportunités sur lesquelles les «néo-entrepreneurs » du secteur se sont appuyés. Pour autant, leurs trajectoires et profils ne sont pas identiques : tous n'ont pas la possibilité d'avoir accès à ces ressources ou la capacité de s'en saisir.

\section{Entre "solaristes" et "néo-artisans" : des profils différenciés}

Au-delà des traits partagés par les néo-entrepreneurs rencontrés, s'observent certaines différences. Celles-ci tiennent à leurs caractéristiques, notamment aux niveaux de diplômes et aux fonctions occupées antérieurement; à la composition et à la conception de l'activité de l'entreprise; aux valeurs et positionnements affichés; aux réseaux dans lesquels ils sont insérés, etc. Deux profils d'entrepreneurs se dessinent : les «solaristes» et les «néo-artisans » ${ }^{(15)}$.

Ici, le capital scolaire et la position professionnelle passée déterminent en partie les ressources des uns et des autres ainsi que leurs capacités à se saisir de nouvelles opportunités pour mettre en place leur projet et déployer leur activité. Ces ressources leur ouvrent en quelque sorte un champ des possibles, par exemple sur ce qui paraît envisageable ou réalisable et sur les moyens disponibles pour atteindre leurs objectifs, qui n'est pas le même dans les deux cas. Elles orientent la façon dont l'activité de l'entreprise est conçue et organisée. Par ailleurs, pour ces nouveaux venus à la fois dans un secteur et une

(14) Pour en savoir plus sur les actions en faveur du développement durable en région Provence-Alpes-Côte-d'Azur, voir le site : http://www.regionpaca.fr/developpement-durable.html ; consulté le 2 juillet 2014.

(15) Cette terminologie est reprise d'une typologie plus large proposée dans Amarillo, LAMANTHE, 2011. 
activité, des processus de construction identitaire sont à l'œuvre qui suivent des voies différenciées : ainsi, ils s'identifient aux groupes professionnels existants ou prennent leurs distances vis-à-vis d'eux de différentes manières selon les individus, de même qu'ils ne s'insèrent pas nécessairement dans les mêmes réseaux d'appartenance.

\section{Capital scolaire et trajectoire antérieure}

Les «solaristes» - Frédéric, Sébastien, François et Pierre - ont un niveau de formation supérieur à celui des «néo-artisans». Ils sont titulaires de diplômes d'ingénieurs et de troisième cycle universitaire (bac +5 , doctorat). Ils ont occupé des postes à haute responsabilité (responsable de la production et directeur adjoint d'une PME, cadre d'une multinationale de l'informatique, directeur technique européen d'un bureau d'études, chef de produit et consultant marketing). Les «néo-artisans» - Denis, Daniel, Éric, Pascal, Philippe, Christophe et Grégory - sont des diplômés de l'enseignement supérieur court (BTS, DUT) ou de niveau bac +4 (maîtrise); seul l'un d'entre eux est de niveau bac +5 (diplômé d'une école de commerce). Ils ont exercé des fonctions engageant plutôt un profil technique (responsable de service, chef d'atelier, responsable de la maintenance, techniciens dans des bureaux d'études, électrotechnicien).

Toutes les entreprises combinent différentes activités au sein même du domaine des énergies renouvelables ou, plus largement, en articulant l'installation d'équipements en énergies renouvelables avec d'autres sources de revenu. Cette polyvalence et cette diversification de l'activité résultent des stratégies mises en œuvre par les dirigeants pour assurer la pérennité de l'entreprise, qu'elles soient pensées d'emblée au moment de la création et/ou qu'elles résultent de réajustements en cours de route (notamment pour faire face à la réduction des aides et incitations publiques). Cependant, les ressorts de ces stratégies, les choix opérés, les possibilités qui sont offertes et les perspectives envisagées par les chefs d'entreprise ne sont pas tout à fait les mêmes pour les deux profils.

Ainsi, les «néo-artisans», s'appuient principalement sur les activités traditionnelles du secteur de la construction, tout en élargissant la gamme de leurs services en proposant l'installation d'équipements en énergies renouvelables.

Daniel réalise des installations en solaire thermique et se spécialise dans les chaudières à granulés bois, qu'il considère comme un créneau encore peu exploré et d'avenir. Dès la création de son entreprise, il a développé une activité de plomberie classique qui l'occupe durant la période pendant laquelle les demandes en chauffage ralentissent. Pascal et Philippe ont, eux aussi, une activité classique de plomberie (neuf, rénovation et dépannage d'urgence) et de chauffagiste (électrique notamment) à laquelle ils associent différents types d'installations (solaire thermique, pompes à chaleur, géothermie, récupération des eaux de pluie). Denis et Daniel ont principalement élargi leur gamme de produits et intégré d'autres sources d'énergies que le solaire: les chaudières à granulés bois représentent $99 \%$ de l'activité du premier, le $1 \%$ restant étant assuré par le solaire thermique; le second combine solaire thermique, photovoltaïque, pompes à chaleur et petit éolien; il y ajoute une activité commerciale en vendant via un site internet différents matériels, essentiellement des diodes électroluminescentes et des ampoules basse consommation. Au moment de l'enquête, Christophe nous précise qu'il a cessé son activité, déclarant qu'il n'a pas été en mesure de faire face à la concurrence d'entreprises à la force de frappe commerciale importante sur le photovoltaïque et à la baisse des aides aux particuliers. Mais s'il a abandonné le photovoltaïque, il n'a pas renoncé pour autant à l'idée de relancer une nouvelle activité basée sur d'autres équipements, comme les pompes à chaleur. Grégory a, quant à lui, décidé de limiter son activité à la pose de panneaux en sous-traitance pour de plus grosses sociétés, arguant des difficultés qu'il a rencontrées pour accéder à une clientèle en propre.

Les activités des «solaristes» sont d'une autre nature, de type conseil, expertise, ingénierie et formation. Frédéric s'est orienté vers la prestation de services auprès des auto-installateurs et des autoconstructeurs, plutôt que vers l'installation stricto sensu. En relation avec un groupement d'achats, il vend du matériel et propose un ensemble de services (conseil, formation à l'équipement et à l'installation, mise en relation avec des installateurs professionnels en photovoltaïque, solaire thermique et poêles à bois). Sébastien se positionne principalement sur l'installation en solaire thermique et propose une activité d'expertise et d'enseignement dans le domaine. François a développé une large gamme d'activités autour de l'ingénierie en solaire photovoltaïque (de l'étude technique jusqu'à l'installation en passant par le montage de dossiers administratifs et financiers), l'expertise, la réalisation d'études, la formation d'installateurs et des activités d'enseignement au Cnam (Conservatoire national des arts et métiers). Pierre combine la conception et l'installation d'équipements en solaire photovoltaïque, de pompes à chaleur, de foyers bois, mais aussi l'isolation par l'extérieur et la pose de planchers chauffants avec une activité de formateur d'installateurs pour Qualit'ENR.

Le positionnement distinct des activités exercées par les «néo-artisans » et les «solaristes» s'explique notamment par leurs capitaux scolaires respectifs : les diplômes plus généralistes et de plus haut niveau (diplômes d'ingénieur, de troisième cycle) des «solaristes» leur permettent d'envisager des activités de conseil, d'expertise et de formation après leur avoir donné les capacités et les compétences 
pour les exercer. Ces activités recoupent les fonctions précédemment occupées dans les entreprises dans lesquelles ils ont travaillé. De telles ressources et activités sont moins accessibles aux «néo-artisans », au profil de technicien plus marqué.

\section{Affichages et positionnements sur le marché}

Ce clivage se retrouve dans la façon dont ils conçoivent et organisent l'activité de leur entreprise, qui obéit non seulement à des différences de constructions identitaires et professionnelles mais aussi à des différences de positionnements au regard de leur nouveau statut, de leur nouvelle activité et sur le marché.

Que ce soit dans les entretiens ou dans la manière dont ils présentent leur entreprise (sur leurs sites internet par exemple), les «solaristes» affichent le solaire et, plus généralement, les énergies renouvelables comme cœur de métier de leur activité. Les valeurs écologiques qu'ils mettent en avant ainsi que leur posture, plus ou moins militante, en faveur des sources d'énergie alternatives, expliquent pourquoi ils ont choisi de créer une entreprise dans ce créneau. Leurs prises de position claires leur permettent également de se différencier de leurs nombreux concurrents - artisans traditionnels et grosses entreprises du bâtiment - et de se faire une place sur un marché nouvellement ouvert.

Frédéric, dont le discours militant fait le lien entre la récupération, le recyclage et l'«état de la planète», a commencé par développer une activité de récupération de déchets (plaquettes de silicium et panneaux photovoltaïques). Son engagement est également visible dans les rapports qu'il entretient avec ses clients et dans la manière dont il gère son entreprise. Par le recours à un groupement d'achats auprès duquel il peut négocier les tarifs, il défend la possibilité d'un accès aux équipements solaires à un prix abordable, soutient l'auto-installation et les autoconstructeurs, les conseille, les forme et affiche un choix «responsable» pour les produits (prix accessibles, fabricants de proximité, fiabilité). Dans son cas, écologie et consommation raisonnée se rencontrent.

Sébastien défend aussi le solaire en tant qu'activité autonome et spécialisée. Il regrette ainsi que dans les appels d'offres concernant le logement collectif, l'installation des équipements solaires soit intégrée aux travaux de plomberie au lieu de figurer comme une spécialité à part entière. Il déclare avoir des positions écologistes "assez extrêmes » : d'après lui, il faudrait faire flamber le prix des énergies fossiles pour que les énergies renouvelables puissent vraiment se développer.

François est sur la même ligne en faisant valoir que le solaire photovoltaïque est une activité spécialisée aux compétences tout à fait spécifiques. Il estime que certains artisans - les électriciens du bâtiment par exemple -, pourtant nombreux à installer ces équipements, n'ont pas les savoir-faire nécessaires. François n'est pas à proprement parler porteur d'un discours écologiste mais il se positionne comme un expert qui met en avant son activité d'ingénierie dans le domaine.

Pierre déclare avoir "une dette envers la planète », qu'il a contractée dans ses activités professionnelles antérieures en prenant fréquemment l'avion. Par conséquent, il a décidé de se mettre en accord avec ses principes, aussi bien dans sa vie personnelle que professionnelle. Il explique ainsi : " J'ai fait ce qu'il fallait pour que la planète se porte mieux», en pratiquant l'écoconstruction chez lui. Il a par exemple installé des chauffe-eaux solaires dans sa résidence principale; ils ne sont certes pas rentables mais représentent un "geste citoyen". Dans son activité, il a souhaité "faire des choses réalisables avec un prix en rapport»; de plus, quand il va voir ses clients, il peut se prévaloir auprès d'eux de ce qu'il a mis en œuvre chez lui.

L'activité des «néo-artisans», quant à elle, s'appuie sur un double positionnement. D'une part, les spécificités techniques propres à l'installation des équipements en énergies renouvelables sont revendiquées et affichées comme cœur de l'activité de l'entreprise (elles en ont justifié le lancement), ce qui permet d'avoir un positionnement concurrentiel clair. D'autre part, elles sont associées aux valeurs traditionnelles de l'artisan : travail bien fait, qualité et maîtrise reposant notamment sur des compétences techniques. L'affichage militant et écologiste est moins présent ici.

Denis se définit à la fois comme technicien et artisan, dans le sens où il déclare qu'il fait tout de $\mathrm{A}$ à $\mathrm{Z}$, refuse la sous-traitance, propose service et disponibilité au client. Il dénonce les abus et l'absence de compétences de certains installateurs.

Éric a eu le projet explicite de devenir artisan; il travaille seul et refuse la sous-traitance. Il affirme ne pas «être piqué écolo» mais être plutôt tourné vers le développement durable. Les énergies renouvelables, "ça lui plaît», et il tente de se démarquer en développant l'installation de chaudières à granulés bois. Sur son site, il se présente comme « un artisan qualifié, qui saura vous conseiller et vous accompagner», qui "guidera simplement vos choix». Il n'est adepte ni du démarchage téléphonique ni des techniques commerciales agressives et met en avant l'argument de la confiance et du conseil personnalisé adapté aux besoins.

Pascal et Philippe déclarent sans ambages que c'est plus la technicité que les perspectives de marché qui leur a plu dans les énergies renouvelables. C'est pourquoi ils se sont d'abord consacrés à la géothermie lorsqu'ils ont lancé leur entreprise. Les deux associés se présentent à la fois comme 
artisans et techniciens (ils se sont "régalés» sur le plan technique avec le photovoltaïque qu'ils ont installé pendant un moment avant de l'abandonner). Les arguments que l'on trouve sur le site de l'entreprise témoignent de leur posture : "ceux qui vous font le devis sont aussi ceux qui vous font l'installation», "le cour de métier est le chauffage».

\section{Réseaux, coopérations et partenariats}

Nouveaux entrants dans l'activité, tous les dirigeants ont été amenés à constituer leurs propres réseaux professionnels et/ou à s'insérer dans des réseaux existants. C'est là qu'ils trouvent en grande partie les ressources qu'ils leur sont nécessaires et qu'ils ne possèdent pas.

Les «néo-artisans» ont rejoint le réseau des organisations professionnelles dédié aux artisans : tous sont adhérents à la Capeb (Confédération de l'artisanat et des petites entreprises du bâtiment) et deux ont souligné le rôle de la Chambre des métiers lors des entretiens. Ils en ont tiré des ressources, sous la forme d'informations, d'aides administratives et de financement des formations (accès au label Qualit'ENR). C'est souvent à l'occasion de ces formations qu'ils ont pu rencontrer d'autres artisans et «néo-artisans» et nouer des relations qui ont pu par la suite déboucher sur des coopérations. Grâce à l'information et à la formation sur leurs produits et aux liens commerciaux qu'ils proposent, les fournisseurs et fabricants de matériel constituent également des ressources non négligeables. Dans la mesure où leurs entreprises ne disposent pas d'une fonction commerciale dédiée, les «néo-artisans» expérimentent différentes solutions : adhésion à une société qui vend des contacts clients établis via un site internet ou partenariat avec une agence immobilière locale qui fait appel à eux pour des travaux.

Les «solaristes» s'insèrent plutôt dans des réseaux de «pairs», i. e. des personnes et organisations avec lesquelles ils partagent des proximités de trajectoires et de démarches. Ils sont aussi à l'origine de la création de leurs propres structures : Frédéric travaille en relation avec un groupement d'achats en direction des autoconstructeurs et auto-installateurs. François s'est inséré dans un réseau d'entreprises formé par des entrepreneurs qui se sont connus en formation, qui consiste concrètement en une association de collaboration et d'entraide "sur la base d'un rapprochement par affinités » (elle leur permet de faire des actions de lobbying auprès d'architectes et de mairies); il s'est lancé dans des actions de partenariat avec des "gens comme lui», des indépendants qu'il a rencontrés dans des formations en bâtiment; il a été membre de la Capeb pendant un temps. Pierre, quant à lui, a fondé un groupement avec une autre entreprise avec laquelle il partage des moyens techniques et humains. Cette solidarité entre "pairs» s'observe aussi dans les pratiques de formation: Pierre a été stagiaire chez un autre «solariste» et lui-même prend en stage des personnes qui souhaitent monter leur propre société. Certains d'entre eux mobilisent fortement les ressources du conseil régional via l'adhésion au Pridès «Bâtiment durable en Méditerranée».

Le positionnement de ces dirigeants concernant l'emploi de salariés est ambigu. Parmi les «solaristes», un seul déclare être employeur: Frédéric a ainsi embauché un ingénieur pour son entreprise d'installation solaire et comptabilise au total quatre salariés dans trois sociétés différentes (solaire et recyclage des déchets). Deux autres «solaristes» ont employé des salariés mais n'en ont plus au moment de l'enquête. Sébastien a été le patron de trois personnes, dont des apprentis, mais il s'en est séparé au moment de la crise de 2008. Pierre a eu deux salariés en contrats à durée déterminée (CDD) qui n'ont pas été reconduits; ils travaillaient dans son entreprise en attendant de créer leur propre activité. Les préférences de ces dirigeants vont plutôt vers des solutions collectives : la coopération (entre ses propres sociétés pour Frédéric), le «système d'entraide entre patrons» (pour Sébastien qui déclare ne plus vouloir embaucher) ou le partenariat «avec des gens comme lui » et des artisans qu'il a formés (pour François), ou encore le groupement d'entreprises (Pierre).

On observe la même réticence du côté des «néoartisans». Denis travaille seul avec sa compagne, à laquelle il s'est associé. Il souhaiterait recruter un technico-commercial en vue de développer une activité de grossiste (poêles à bois) et prévoit d'embaucher un de ses anciens collègues de travail avec lequel il est devenu ami. Il fait sinon appel à un autre artisan en cas de besoin. Éric travaille seul et sollicite de même d'autres artisans. Pascal et Philippe travaillent à deux et disent ne pas avoir les moyens d'embaucher car "c'est trop cher»; ils déclarent d'ailleurs ne pas se verser de salaire de façon régulière. Seul Daniel est employeur : il déclare préférer avoir des salariés plutôt que de coopérer avec d'autres artisans dont il n'est pas sûr des pratiques. Sur ce plan, les «néo-artisans» ne sont pas différents des «solaristes».

Les réticences à embaucher s'expliquent notamment car les dirigeants craignent de ne pas être en mesure d'assurer un salaire tous les mois, ce qui est révélateur des difficultés que plusieurs rencontrent pour assurer une activité régulière à leur entreprise. L'activité est au contraire volatile, comme en témoignent les obstacles que nous avons dû surmonter pour contacter les entreprises. Dans la mesure où un certain nombre d'entreprises sont restées injoignables (au total huit), nous avons supposé qu'elles avaient cessé leur activité; ce chiffre estimé ajouté aux cessations que nous avons effectivement constatées représente une proportion relativement importante des entreprises du secteur. De plus, huit entreprises toujours en activité ont arrêté le solaire. 
Ce sont principalement des entreprises traditionnelles du bâtiment (maçonnerie, électricité générale, chauffage, plomberie) qui ont tenté de se lancer dans le solaire (essentiellement le photovoltaïque) pour compléter leur activité. Seule une entreprise créée par un des «néo-entrepreneurs» rencontrés a cessé son activité (Christophe).

On peut faire l'hypothèse que les «néo-entrepreneurs» ont fait preuve de davantage de capacités de résistance et de facultés à se maintenir durablement sur le marché des énergies renouvelables que les artisans traditionnels, notamment à partir du moment où l'appel d'air apporté par les incitations fiscales s'est fortement ralenti. On peut imaginer que leur niveau de formation et les compétences acquises dans les postes occupés antérieurement leur ont permis de mieux s'en sortir, ne serait-ce que parce qu'ils ont été plus en mesure que les artisans traditionnels de mettre en place et d'assurer un ensemble de tâches et de services au-delà de la seule installation (comme le montage des dossiers de demande d'aide pour les clients, la conception de travaux d'ensemble, le calcul des performances des équipements, etc.). Sur ce point comme sur d'autres, de nouvelles investigations semblent nécessaires pour voir, dans la durée, comment ont évolué les trajectoires professionnelles de ces salariés devenus «néo-entrepreneurs» suite aux restructurations financières des années 2000.

\section{Bibliographie}

Amarillo H. (2009), L'enjeu des qualifications face au défi énergétique et environnemental dans la construction, Rapport pour la CGT, Aix-en-Provence, Lest.

Amarillo H., Lamanthe A. (2011), The social construction of economic activities: entrepreneurs, skills and accreditation in the French solar energy sector, 27th EGOS Colloquium, Gothenborg, 7-9 juillet.

Beaujolin-Bellet R., Lerais F., Paucard D. (2012), «Introduction. Les modes de gestion des restructurations : quoi de neuf ?», Revue de l'Ires, n ${ }^{\circ}$ 72, pp. 3-28.

BOURDieu P. (2000), Les structures sociales de l'économie, Paris, Seuil.

Bourguignon R., Garaudel P. (2012), «Les départs volontaires comme mode de sélection des salariés licenciés: des risques à maîtriser", Revue de l'Ires, $\mathrm{n}^{\circ} 72$, pp. $91-114$.

Colombard-Proust M., Laumonier C., Roudil N. (2007), Artinova. Le rôle des artisans dans la diffusion des meilleures techniques énergétiques disponibles, Rapport final, Centre scientifique et technique du bâtiment (CSTB), octobre; disponible en ligne à l'adresse : http://www.cstb.fr/fileadmin/documents/ webzines/2008-02/Rapport_Artinova_nov_2007.pdf; consulté le 3 juillet 2014 .

Conseil d'orientation POUR L'EMPloi (2010), Croissance verte et emploi, rapport pour le ministère de l'Écologie et du Développement durable, 25 janvier, Paris, Conseil d'orientation pour l'emploi; disponible en ligne à l'adresse : http://www.ladocumentationfrancaise.fr/var/ storage/rapports-publics/104000053/0000.pdf; consulté le 3 juillet 2014.

Darbus F. (2008), «L'accompagnement à la création d'entreprise. Auto-emploi et recomposition de la condition salariale», Actes de la recherche en sciences sociales, $\mathrm{n}^{\circ} 175$, pp. 18-33.
Fligstein N. (2005), « States, markets and economic growth », in Nee V., Swedberg R. (Ed.), The economic sociology of capitalism, Princeton and Oxford, Princeton university press, pp. 119-143.

Garnier J., Lamanthe A., Lanciano-Morandat C., Mendez A., Mercier D., Rychen F. (2004), Les modes de transition du tissu productif régional en Provence-AlpesCôte-d'Azur. Une étude comparative de quatre sites, Aix-en-Provence, Lest; disponible en ligne à l'adresse : http://halshs.archives-ouvertes.fr/docs/00/08/63/37/ PDF/modestrans.pdf; consulté le 3 juillet 2014.

Gallenga G., Lamanthe A. (2012), La question énergétique comme analyseur de la reconversion d'un territoire, rapport pour l'OHM du bassin minier de Provence/CNRS INEE, Aix-en-Provence, Lest.

Garnier J., Lanciano-Morandat C. (2004), «Le bassin minier de Provence, territoire incertain, transition inachevée», in Garnier J. et al., Les modes de transition du tissu productif régional en Provence-Alpes-Côte-d'Azur. Une étude comparative de quatre sites, Aix-en-Provence, Lest, pp. 185-261.

Hirschman A.-O. (1972), Face au déclin des entreprises et des institutions, Paris, Éditions ouvrières.

Kerbourc'H J.-Y. (2007), «L'anticipation des restructurations à l'épreuve du droit du travail», Travail et emploi, $\mathrm{n}^{\circ} 109$, pp. 25-37.

Lamanthe A. (2011), Gens de métiers, acteurs du territoire, Tome 2: «Le rôle des qualifications dans les dynamiques économiques de trois territoires: Pays d'Aubagne, Grand Avignon, Briançonnais», Aix-enProvence, Marseille, Observatoire régional des métiers (ORM).

Legros S. (2009), «Artisans atypiques: une invasion bénéfique ou périlleuse ?», Métiers Atlantique, n 85, pp. 14-20; disponible en ligne à l'adresse : http://www. cma-nantes.fr/portals/65/basedoc/Communication/ 
Dossier_Metiers_Atlantique/Dossier_85_artisans atypiques.pdf; consulté le 3 juillet 2014.

Mazaud C. (2012), «Artisan, de l'homme de métier au gestionnaire?», Travail et emploi, $\mathrm{n}^{\circ}$ 130, pp. 9-20.

Moreau M.-A. (2007), «Introduction. Dossier : Restructurations», Travail et emploi, ${ }^{\circ}$ 109, pp. 7-9.

Pochic S. (2001), «La menace du déclassement. Réflexions sur la construction et l'évolution des projets professionnels de cadres au chômage», Revue de l'Ires, $\mathrm{n}^{\circ} 35$, pp. 61-88.
RAVEYRE M. (2005), «Introduction. Les restructurations : vers un état d'instabilité durable ?», Revue de l'Ires, $\mathrm{n}^{\circ} 77$, pp. 7-17.

Testenoire A. (2000), «Un compromis de coexistence : négociation conjugale des trajectoires professionnelles », in Bertrand H., Degenne A., Guillot O. (ed.), Trajectoires d'emploi et conditions d'existence des individus, Marseille, Céreq, coll. «Documents», «Série Séminaires », $\mathrm{n}^{\circ}$ 148, pp. 107-119.

Zimmermann B. (2011), Ce que travailler veut dire. Une sociologie des capacités et des parcours professionnels, Paris, Economica. 